\title{
Magnetic Resonance Imaging-based Radiomics Nomogram for Prediction of the Histopathological Grade of Soft Tissue Sarcomas: A Two-center Study
}

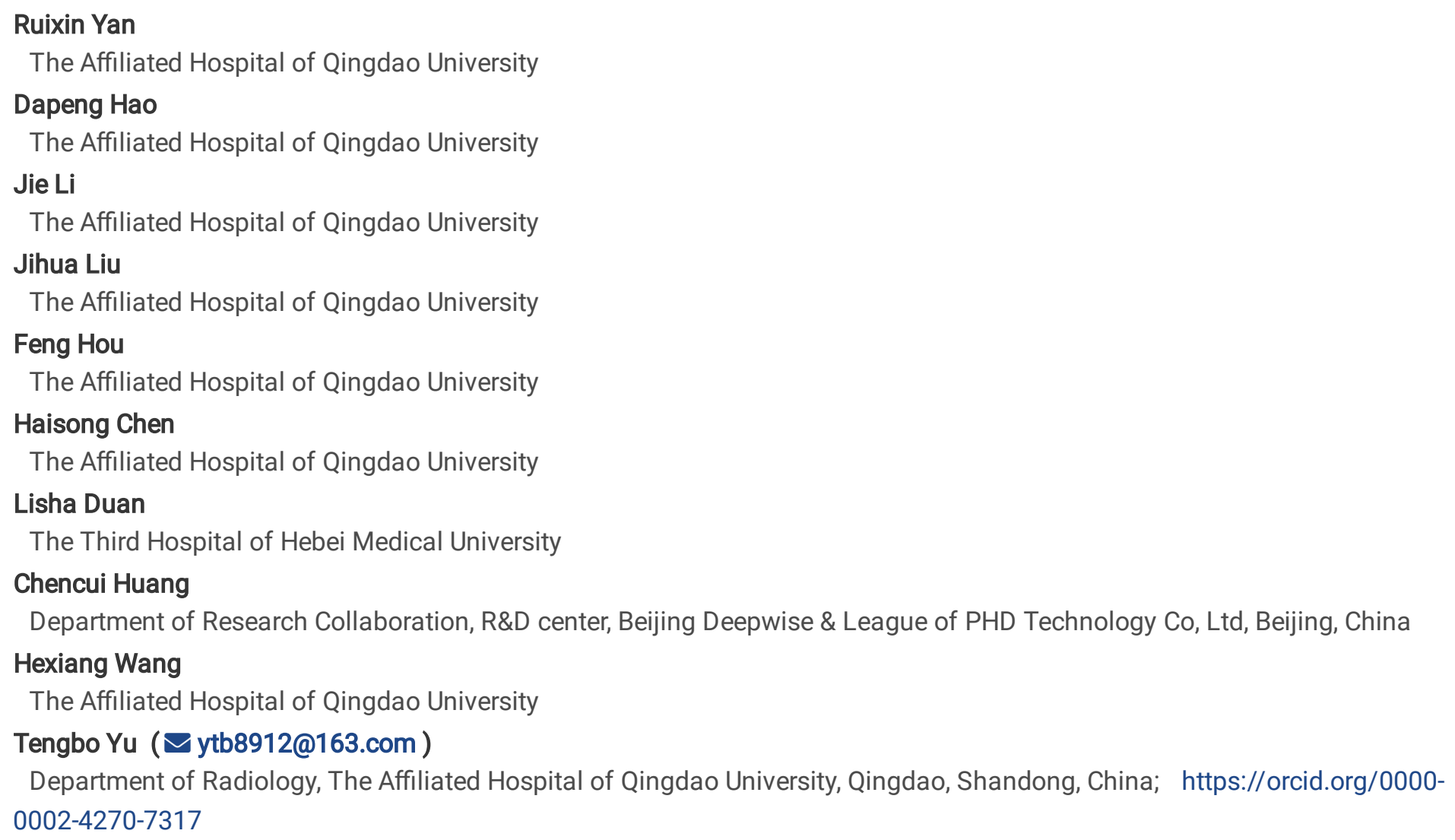

\section{Research}

Keywords: Magnetic resonance imaging, Soft tissue sarcomas, Radiomics, Tumor grading

Posted Date: October 9th, 2020

DOI: https://doi.org/10.21203/rs.3.rs-87126/v1

License: (c) (i) This work is licensed under a Creative Commons Attribution 4.0 International License. Read Full License

Version of Record: A version of this preprint was published at Journal of Magnetic Resonance Imaging on February 18 th, 2021. See the published version at https://doi.org/10.1002/jmri.27532. 


\section{Abstract}

Background: Preoperative prediction of the soft tissue sarcomas (STSs) grade is important for treatment decisions. To preoperatively distinguish low-grade (grades I and II) and high-grade (grade III) STSs, we developed and validated the performance of a magnetic resonance imaging (MRI)-based radiomics nomogram.

Methods: Patients with an STS based on the French Federation of Cancer Centers Sarcoma Group grading system at two independent institutions were enrolled (training set, $n=109$; external validation set, $n=71$ ). The minimum redundancy maximum relevance method and least absolute shrinkage and selection operator logistic regression were used to process feature selection and radiomics signature development. Three radiomics signature models were constructed based on T1weighted imaging (RS-T1 model) and fat-suppressed T2-weighted imaging sequences (RS-FST2 model) and their combination (RS-Combined model). Model performance (discrimination capability, calibration curve, and clinical usefulness) was evaluated in the external validation set.

Results: The RS-T1 model, RS-FST2 model, and RS-Combined model achieved predictive abilities with area under the receiver operating characteristic curves (AUCs) of $0.645,0.641$, and 0.829 , respectively, in the external validation set. The nomogram, incorporating significant clinical factors and the RS-Combined model, showed extremely high predictive ability in the training set and external validation set with AUCs of 0.916 (95\% confidence interval, 0.866-0.966) and $0.879(0.791-0.967)$, respectively. The nomogram achieved significant patient stratification.

Conclusions: The proposed noninvasive MRI-based radiomics nomogram shows superior predictive performance in differentiating low-grade from high-grade STS.

\section{Background}

Soft tissue sarcomas (STSs) are a heterogeneous group of malignant mesenchymal neoplasms [1]. They account for $1 \%$ of all tumors and have a high mortality rate of about 50\% [2]. According to the American Cancer Society, the incidence of STS was approximately 8790 cases each year in the United States in 2017 [2]. This is comparable with the annual occurrence of esophageal cancer (17,500 cases) and cervical cancer (12,000 cases) [3]; thus, the rarity of STS may be overestimated. Surgery is considered the cornerstone curative treatment for localized STS with or without radiation therapy, and chemotherapy can be added for selected patients. However, survival remains unsatisfactory; tumor recurrence is common, and the 5-year survival rate is approximately $50 \%-60 \%[4,5]$. Therefore, identification of prognostic biomarkers is needed to stratify high-risk patients and improve the prognosis.

The histopathological grade is considered the most significant prognostic factor in making optimal clinical therapy decisions for patients with STS [6]. STSs can be defined as grades I to III according to the French Federation of Cancer Centers Sarcoma Group (FNCLCC) grading system, which is based on histologic tumor characteristics such as the mitotic count, tumor differentiation, and necrosis [7]. The prognosis of high-grade (grade III) STSs may improve with (neo)adjuvant chemotherapy, while low-grade STSs (grades I and II) can avoid the potential unnecessary treatment [8-10]. The preoperative histological tumor grade is mainly confirmed through needle biopsy [11]; however, the pathological results are closely related to the sample size, tumor site, and internal components of the tumor. Additionally, because of tumor heterogeneity, the initial biopsy grade sometimes underestimates the actual grade of the surgical specimen $[7,12]$. Consequently, formulation of an accurate and noninvasive preoperative STS grading method is essential to identify patients who are most likely to benefit from optimal adjuvant treatment at the earliest possible time.

Magnetic resonance imaging (MRI) is integral to the detection, assessment, diagnosis, and follow-up of STS because of its excellent soft tissue contrast resolution and noninvasiveness. Previous studies have revealed specific conventional MRI features that are associated with the tumor grade $[13,14]$. However, direct voxel-to-voxel contrast of histologic grades and imaging features is lacking [6]. Therefore, the STS grade cannot be determined by MRI alone. 
Radiomics has the capability to express intratumoral heterogeneity in a noninvasive manner with large-scale digital medical images using high-throughput extraction of innumerable quantitative features [15]. Moreover, radiomics provides comprehensive knowledge because the data are obtained from the entire tumor instead from just a focal sample [16]. Furthermore, radiomics is reproducible. Thus, MRI-based radiomics would be broadly applicable to patients with sarcomas. Radiomics has been successfully applied in prediction of the histologic grade, local recurrence or distant metastasis, overall survival, and response to neoadjuvant therapy in patients with STSs [17-22]. Most previous reports defined high-grade STS as grades II and III, while we define high-grade STS as grade III based on recently published studies [6, 22]. Additionally, the use of MRI-based radiomics for prediction of high-grade STS (grade III) has not been widely recognized and requires further validation. Moreover, MRI-based radiomics nomograms that combine radiomics and clinical factors for STS grading using data from multiple centers are relatively limited.

This study was performed to develop an MRI-based radiomics nomogram using a two-center dataset and determine whether the nomogram can distinguish the preoperative grade of STS. Additionally, a radiomics nomogram-based model was generated for prognostic evaluation. Patient risk stratification and clinical decision benefits were analyzed for the established model in an effort to improve personalized clinical treatment strategies and therapeutic decisions.

\section{Methods}

\section{Data collection}

Ethical approval for this two-center retrospective study was provided by our hospital's institutional review board, which waived the requirement for informed consent. Pre-therapeutic T1-weighted imaging (T1WI) and fat-suppressed T2-weighted imaging (FS-T2WI) of 180 patients with STS were retrospectively analyzed. All patients' FNCLCC tumor grades had been pathologically confirmed using postoperative specimens from November 2007 to November 2019. The minimum follow up time is 5 mouths. Detailed descriptions of the inclusion and conclusion criteria are shown in Supplementary Material. The patients' data were gathered from The Affiliated Hospital of Qingdao University (training set, $n=109$ ) and The Third Hospital of Hebei Medical University (external validation set, $n=71$ ). The final pathologic results of the 180 patients with STS are shown in Table 1.

The patients were subsequently grouped into a low-grade group $(n=93)$ and high-grade group $(n=87)$ according to their FNCLCC tumor grade. The patients' basic data are shown in Table 2. The patients comprised 112 male and 68 female patients with a mean age of 52.4 years (range, 1-93 years). Fig. 1 shows a flow chart of the enrolled patients and radiomics implementation.

\section{MRI acquisition and region-of-interest segmentation}

All 180 patients underwent MRI scanning using a GE MRI 1.5T, GE Signa HDx 3.0T (GE Medical Systems, Milwaukee, WI, USA), Siemens Skyra 3.0T, Siemens Magnetom Prisma 3.0T (Siemens Healthcare GmbH, Erlangen, Germany), or Philips Achieva 1.5T (Philips Medical Systems, Best, the Netherlands). The following scanning parameters were used: T1WI (repetition time [TR] / echo time [TE], 420-680 ms / 6.1-20 ms); FS-T2WI (TR / TE, 2640-5000 ms/ 30-102 ms); section spacing, 1 mm; section thickness, 3-4 mm, matrix, $320 \times 320$; field of view, 200-400 mm Three-dimensional region of interest (3D-ROI) segmentation of all tumors was conducted manually using ITK-SNAP open-source software (v.3.8.0; http://www.itksnap.org). The ROI was outlined according to the contour of the tumor from each transverse layer on preoperative T1WI and FS-T2WI sequences and automatically turned into a 3D-ROI. The 3D-ROI segmentation covered the entire primary tumor and avoided obvious peritumoral edema. Intraobserver and interobserver intraclass correlation coefficients (ICCs) were calculated to test the intraobserver reproducibility and interobserver reliability for the radiomic feature extraction of 40 random patients. Readers 1 and 2 drew the 3D-ROIs, and the next Reader 1 repeated the segmentation after 1 month. The ROI segmentation depicted by Reader 1 were used for further analysis. Intraobserver and interobserver ICCs of $>0.75$ were included for the subsequent investigation.

\section{Image preprocessing and radiomics feature extraction}


Preprocessing procedures were applied to compensate for inhomogeneous intensity caused by different institutions and to decrease the variability of features. A method to decrease the number of gray levels and thus improve the signal-to-noise ratio of the texture calculations results was applied. The 3D-ROIs were then isotropically resampled to a planar resolution (voxel size $=1$ $\times 1 \times 1 \mathrm{~mm}^{3}$ ) using cubic interpolation to standardize the voxel spacing $[23,24]$.

3D Slicer software (v.4.10.2; https://www.slicer.org/) was implemented for radiomics feature extraction. Using this software, a range of radiomics features was extracted and the intratumoral heterogeneity of the segmented 3D-ROls was quantitatively expressed by the extracted features. The radiomics features $(n=1130)$ were respectively derived from T1WI and FS-T2WI sequences from each 3D-ROI, incorporating shape features, first-order features, texture features including the gray-level cooccurrence matrix, gray-level dependence matrix, gray-level size-zone matrix, gray-level run-length matrix, and neighboring gray tone matrix and wavelet decomposition features.

\section{ComBat compensation method}

Effects obtained by different MRI scanners and protocols were removed using the ComBat compensation method while retaining its outperforming features in texture patterns, which potentially improved the power and reproducibility of subsequent investigations $[25,26]$.

\section{Patients' clinical data and MRI features}

Clinical data (age, sex, and TNM stage) and MRI features were analyzed. The TNM stage was determined using the preoperative MRI and computed tomography information. Each musculoskeletal MRI scan was evaluated by two readers who had 7 and 14 years of experience and were blinded to the clinical and histopathological data. A consensus was reached in cases of discrepancy. The recorded data were described in Supplementary Material.

A pathologist (F.H.) with 11 years of experience in soft tissue diseases explained the pathology, incorporating the stage and histologic subtype. The FNCLCC system assigns a score for the tumor's mitotic index, differentiation, and extent of necrosis, and the tumor grade is obtained by summing these three scores. The pathologic TNM stage was determined based on the guidelines in the American Joint Committee on Cancer (AJCC) Cancer Staging Manual, 8th edition.

\section{Construction of radiomics signature}

A subsequent statistical analysis was performed using R software (v3.5.1; https://www.R-project.org). After removing redundant and irrelevant features and retaining the most related features for grading of STS by applying the minimum redundancy maximum relevance algorithm, the 30 best radiomics features were selected and applied to least absolute shrinkage and selection operator (LASSO) regression to generate the radiomics signature[27]. Next, the radiomics features with non-zero coefficients selected from LASSO regression formed three radiomics signatures based on T1WI sequences (RS-T1 model), FST2WI sequences (RS-FST2 model), and their combination (RS-Combined model). The radiomics score (rad-score) was calculated according to its linear combination of corresponding LASSO non-zero coefficients.

\section{Development of clinical model and radiomics nomogram}

Univariate logistic regression was performed for the clinical risk factors and MRI features used to evaluate the STS grade. The factors with a two-sided $P$ value of $<0.05$ were then introduced into a multivariate logistic regression. The variables with a twosided $\mathrm{P}$ value of $<0.05$ from the multivariate analysis were considered potential independent clinical risk factors associated with the histologic grade and were used to compose a clinical model. Ultimately, a clinical model was established. Finally, the significant clinical factors and the optimal radiomics signature were selected and combined in the radiomics nomogram.

\section{Validation of the radiomics nomogram and performance assessment of differentmodels}

The radiomics nomogram was assessed for discrimination, calibration, and clinical application [28] in both sets. The discrimination capability of the clinical model, radiomics signatures models and radiomics nomogram to correctly distinguish the grade was quantified by the AUC and accuracy. The Hosmer-Lemeshow test was used to assess the goodness-of-fit with a

Page $4 / 20$ 
calibration curve to evaluate the calibration of the nomogram [29]. The external validation set was used to test the radiomics nomogram, and the rad-score was correspondingly calculated using the formula established in the training set.

The AUC between each two of the three models was evaluated using the DeLong test. The clinical application was estimated by a decision curve analysis (DCA) to determine whether the radiomics nomogram can be regarded as robust and effective. The DCA was used to quantitatively calculate the net benefits for a range of different threshold probabilities in the whole cohort.

\section{Follow-up and survival analysis}

The patients underwent MRI or computed tomography follow-up examinations every 6 to 12 months for the first 2 years and annually thereafter. Progression-free survival (PFS) was calculated as the survival endpoint for patient outcomes from the time of surgery to the time of radiographic detection of recurrence, time of last follow-up examination, or time of death without evidence of progression. Patients were censored in case of on 30 November 2019.

Survival curves were generated based on Kaplan-Meier survival analysis. Differences in survival curves were assessed by the log-rank test. The pathologic grade results model, radiomics signature model, and radiomics nomogram model were further evaluated for their performance in PFS stratification. We combined the nomogram model with the AJCC staging system (Cancer Staging Manual, 8th edition) to analyze its ability in PFS stratification.

\section{Statistical analysis}

$\mathrm{R}$ software was used to perform the statistical analysis. A two-sided $\mathrm{P}$ value of $<0.05$ was regarded as statistically significant.

A univariate analysis was performed to evaluate the relationships between the patients' characteristics. For continuous variables, Student's t-test or the Mann-Whitney U test was used to determine whether significant inter-group differences existed between the low-grade and high-grade groups; for classified variables, the chi-square test or Fisher's exact test was performed where appropriate. The packages we used in R software were described in Supplementary Material.

\section{Results}

\section{Clinical factors and modeling}

The basic clinical characteristics of the 180 patients with STS are shown in Table 2. The T-stage, MRI-reported margin, and median PFS were significantly different between the low-grade and high-grade groups in both the training set and external validation set (both $P<0.05$ ). The remaining factors showed no significant difference between the two groups in either the training set or external validation set. The results of the univariate and multivariate logistic regression analyses with $P<0.05$ are shown in Table 3. Based on the results of the multivariate logistic regression analysis, the T-stage and MRI-reported margin were included to create a clinical model, with an AUC of 0.787 and 0.833 in the training set and external validation set, respectively. The results are shown in Table 4 and Fig. 3.

\section{Radiomic feature selection and radiomic signature performance}

Both intraobserver and interobserver ICCs of $>0.75$ were considered features with robustness and were included in the subsequent investigation. The selected radiomics features of the RS-Combined model are shown in Fig. 2a. The corresponding rad-score of each patient is shown in Fig. 2b and c. The AUC was significantly different between the RS-Combined model and RS-FST 2 model $(P<0.05$ both in the training set and external validation set). The AUC showed significant difference between the RS-Combined model and RS-T1 model $(P<0.001)$ or RS-T1 model and RS-FST2 model $(P<0.001)$ in the external validation set. The AUC showed no significant difference between each two of the following models in the training set: RS-Combined model vs. RS-T1 model, $\mathrm{P}=0.297$ and RS-T1 model vs. RS-FST2 model, $\mathrm{P}=0.076$. The RS-Combined model was chosen for construction of the radiomics nomogram because it had the highest AUC in both sets. The predictive performance of these models is shown in Table 4 and Fig. 3.

\section{Patient risk stratification andvalidation of the radiomics nomogram}


Kaplan-Meier survival curves of the different models are shown in Fig. 4. The stratified analysis is shown in Supplementary Material. A radiomics nomogram that combined the RS-Combined model and significant clinical factors was subsequently constructed (Fig. 5a). The predictive performance of these models is shown in Table 4 and Fig. 3. The AUC showed a significant difference between the Nomogram model and Clinical model $(P<0.05)$ and Nomogram model and RS-Combined model $(P<$ $0.05)$ in the training set. The AUC showed no significant difference between each two of the following models: RS-Combined model vs. Clinical model, $\mathrm{P}=0.349$ and 0.965 in the training and external validation set, respectively; Nomogram vs. RSCombined model, $\mathrm{P}=0.209$; and Nomogram vs. Clinical model, $\mathrm{P}=0.388$ in the external validation set. Kaplan-Meier curves showed that the nomogram-predicted model significantly stratified patients for PFS in both sets $(\log$ rank $P<0.05$, respectively; Fig. 4k, I).

The calibration curve of the radiomics nomogram showed good agreement between the predicted and observed tumor grade in both sets (Fig. 5b, c.). The Hosmer-Lemeshow test result was not statistically significant $(P=0.872$ in the training set and $P=$ 0.506 as verified by the external validation set). The DCA indicated that the radiomics nomogram had a higher overall net benefit than the radiomics signature and the clinical model in predicting the preoperative STS grade considering the "treat all" and "treat none" schemes through the reasonable treatment threshold probability (Fig. $5 \mathrm{~d}$ ), proving its clinical usefulness.

\section{Prognostic radiomic prediction models show moderate performance}

We also assessed whether the established models offer increased benefits over the clinical staging system. The radiomics nomogram model achieved optimal predictive performance for PFS in the external validation set with a concordance index (Cindex) of 0.584 [95\% confidence interval $(\mathrm{Cl}), 0.511-0.658$ ] compared with the RS-T1 model (C-index: $0.568,95 \% \mathrm{Cl} 0.492-0.641$ ), RS-FST2 model (C-index: 0.507, 95\% Cl 0.424-0.589), RS-Combined model (C-index: 0.564, 95\% Cl 0.487-0.641), and the AJCC staging system alone (C-index: $0.526,95 \% \mathrm{Cl} 0.450-0.601)$.

Finally, the model that combined radiomics nomogram model with the best-selected predictive performance and the AJCC staging system achieved a slightly increased benefit (C-indices for radiomics nomogram + AJCC: 0.591, 95\% Cl 0.492-0.641).

Furthermore, the radiomics nomogram combined with the AJCC staging system significantly stratified patients for PFS (log rank $P<0.010$; Fig. 5e).

\section{Discussion}

Preoperative grading of STS is critical for choosing the optimal treatment method (neoadjuvant treatment or radiation therapy). Additionally, preoperative grading has been proven to independently affect the prognosis in patients with STS [6, 30]. However, the preoperative grade based on biopsy examination results is sometimes underestimated because of tumor heterogeneity [7]. In this study, a noninvasive method derived from massive clinical and MRI data was investigated for predicting the preoperative histologic grade. The radiomics nomogram, which combined the RS-Combined model with clinical factors, successfully distinguished high-grade from low-grade STS with the highest performance and exhibited good calibration in both sets, indicating the incremental value of the nomogram and showing that it can be a promising tool for clinical strategy adjustment. Additionally, the developed radiomics nomogram model significantly stratified patients into low- and high-risk patients for PFS. The model that combined the radiomics nomogram and the AJCC staging system showed better prognostic ability than the AJCC staging system alone with significant patient risk stratification. The radiomics models showed moderate performance for the prognostic prediction of PFS.

MRI is widely used for characterization of soft tissue tumors. Previous reports have noted some qualitative MRI characteristics that may serve as potential imaging biomarkers of the histopathologic STS grade. Zhao et al. [14] found that high-grade STS tended to have high peritumoral signal intensity on T2-weighted images. Crombé et al. [6] confirmed that MRI features of necrosis, heterogeneity, and peritumoral enhancement are associated with high-grade STS. In this study, a clinical model that included the T-stage (which represents tumor size) and MRI-reported margin was proven to predict the tumor grade, which is partly consistent with recent reports $[13,14]$. The results indicated that patients with high-grade STS are more likely to have an ill-defined margin, and one reason may be that high-grade tumors show greater invasive ability in peripheral tissues [14]. 
However, although traditional MRI interpretation reflects the macroscopic imaging features, it lacks objectivity and quantification and tends to be influenced by the radiologist's experience.

Radiomics as an emerging favorable alternative method for preoperatively describing the quantitative characteristics of tumors. It produces maximized information from large-scale radiologic images that is beyond qualitative evaluation by visual inspection [31-33]. Therefore, radiomics based on MRI scans may achieve better decision-making. Corino et al. [34] reported that a radiomics classifier based on apparent diffusion coefficients in 19 patients could be used to distinguish grade II from III STS. Xiang et al. [35] found that quantitative MRI-based histogram parameters can differentiate the grade of STS, Zhang et al. [17] demonstrated that FS-T2WI-based radiomics could be used to predict the histopathological grade of STS in a study with a small cohort of 35 patients, and Wang et al. [18] found that radiomics signature-based machine-learning classifiers can distinguish low-grade from high-grade STS. Nevertheless, these studies had small sample cohorts and two of them lacked a validation set, which potentially lead to the problem of overfitting. Conversely, we defined high-grade STS as grade III with balanced patient data, retrospectively enrolled more patients from two different hospitals, combined a clinical model to form a radiomics nomogram, and validated model efficiency in an external validation set. Additionally, conventional T1WI and FS-T2WI examinations were used for radiomics feature extraction because these techniques are commonly used, easy to access, familiar among radiologists, and show stability in clinical practice. Conversely, multi-modality MRI sequences such as diffusionweighted imaging readily exhibit distortion and susceptibility artifacts, and dynamic contrast-enhanced images tend to be influenced by the injected contrast medium [36].

The RS-Combined model established in this study showed good reproducibility with a similar AUC and accuracy in the training set and external validation set, indicating that RS-Combined model was generalizability and stability. Peeken et al. [37] established a radiomics-combined model based on contrast-enhanced T1-weighted fat-saturated (T1FSGd) and fat-saturated T2-weighted (FS-T2WI) MRI sequences with an AUC of 0.76. However, because most patients in our study underwent noncontrast-enhanced T1WI and FS-T2WI examinations, we could only construct a more generalized RS-Combined model based on T1WI and FS-T2WI, and it achieved an AUC of 0.829 in the external validation set and showed prediction performance similar to that reported by Peeken et al. [37]. This might indicate that good model performance can also be obtained by incorporating T1WI instead of T1FSGd in future studies. This will allow patients to avoid invasive procedures and the potential risks of contrast-enhanced examinations, such as contrast agent allergy and increased liver and kidney metabolic burdens.

We established a radiomics nomogram that combined independent clinical factors and the RS-Combined model, showing the best AUC in each dataset, better calibration, and highest net benefit in a range of threshold probabilities. This is consistent with recent reports of stratification of patients with glioblastoma and soft tissue tumors [37,38]. The nomogram graphically creates a clinical statistical predictive model, is easy to use, and enables accurate prediction of an individual patient's probability of preoperative stratification. The preoperative nomogram model allowed us to stratify patients, identify patients with high-grade STS who may need adjuvant systemic therapies, and more confidently establish a rational follow-up schedule [39, 40]. The nomogram can be applied in different clinical situations to provide complementary staging information, such as when a biopsy specimen is difficult to access anatomically or when the biopsy result is unclear.

We also assessed the prognostic ability of the developed radiomics models. Radiomics models solely developed to predict PFS displayed only moderate predictive ability. The radiomics nomogram grading model significantly stratified patients into low- and high-risk groups in both the training and external validation sets compared with the other models. The radiomics nomogram showed a slightly enhanced prognostic capacity when combined with the AJCC staging system.

Previous investigations have demonstrated that radiomics can be used to predict survival outcomes in patients with STS. Spraker et al. [20] found that radiomics features alone or combined with age and grade were respectively independent predictors of over survival, and the latter achieved best performance. Peeken et al. [37] demonstrated that the radiomics model based on T1FSGd MRI sequences showed significant patient stratification performance for overall survival, and improved prognostic performance was found with the combination of a T2FS radiomics model with the AJCC system. Conversely, we evaluated survival prediction by incorporating a nomogram-predicted grade model that included clinical stage and MRI features, and it showed superior patient risk stratification performance. Unlike Peeken et al. [37], we found that the pathologic-reported grade

Page $7 / 20$ 
model itself achieved significant stratification performance, making it easy for the proposed radiomics nomogram models to be potential surrogate markers. However, the radiomics nomogram models significantly stratified patients in the training set only by a close margin $(P=0.047)$. Additionally, the radiomics nomogram combined with the AJCC staging system generated more significant PFS stratification performance than the nomogram and AJCC staging system alone. The filter models might show promise for the long-term management of patients with STS.

Our study had several limitations. First, we retrospectively examined the images; thus, selection bias was inescapable despite our strict criteria. Second, manual tumor segmentation by a team may lead to irregularities. Key techniques such as automatic segmentation may be more accurate and time-saving and can be used to improve the robustness of radiomics models in future research. Third, our data were obtained from two institutions that used similar but different scanners and protocols. Therefore, resampling methodology and the ComBat compensation method were applied to reduce the differences in image specifications, aiming to increase the stability of features and different models. Finally, sample enlargement is needed in future studies.

\section{Conclusion}

In conclusion, we identified and validated a noninvasive radiomics nomogram combined with the radiomics signature and clinical factors. It exhibited satisfactory predictive performance in differentiating the preoperative histopathological grade of STS and achieved superior patient risk stratification performance. We expect that these results will help to improve clinical treatment strategies and improve survival outcomes in selected patients.

\section{List Of Abbreviations}

3D-ROI: three-dimensional region of interest; ADC: apparent diffusion coefficient; AJCC: American Joint Committee on Cancer; Cl: confidence interval; CT: computed tomography ; DCA: decision curve analysis ; FNCLCC: the French Federation of Cancer Centers Sarcoma Group; FOV: field of view; FS-T2WI: fat-suppressed-T2 weighted images; ICCs: intra-observer and inter-observer correlation coefficients; LASSO: least absolute shrinkage and selection operator; MRI: magnetic resonance imaging; mRMR: minimum redundancy maximum relevance; ROC-AUC: area under the receiver operating characteristics (ROC) Curve; ROI: region of interest; RS-T1 radiomics signature T1Wl; RS-FST2: radiomics signature FS-T2WI; RS-Combined: radiomics signature combined; SNR: signal-to-noise ratio; STS: soft tissue sarcoma; TR: repetition time; T1WI: T1-weighted images; TE: echo time

\section{Declarations}

\section{Ethics approval and consent to participate}

Our Institutional Review Board (Medical Ethics Committee of the Affiliated Hospital of Qingdao University) approved this study and waived the need for informed consent from patients.

\section{Consent for publication}

All authors have read and approved the content and agree to submit for consideration for publication in the journal.

\section{Availability of data and materials}

All data generated or analysed during this study are included in this published article.

\section{Competing interests}

The authors declare that they have no competing interests.

\section{Funding}

This study has received funding by the National Science Foundation for Young Scientists of China (Grant No. 81571673区 81671658); This work was supported by the Medical and health science and Technology Development Plan of Shandong 
Province. Grant no. 2019WS373.

Author Contributions

All authors have had access to the data and all drafts of the manuscript. Specifc contributions are as follows: design and supervision: $H D, L J, W H, C H$; development of methodology: $L J, H D, W H$; collection and extraction of data: $D L, H C, H F, Y R$; analysis and interpretation of data (e.g., statistical analysis, algorithm and software development): LJ, WH, YR; manuscript writing: WH, YR; final approval of manuscript: all authors.

\section{Acknowledgements}

We appreciate the help from other teammates.

Authors' information

1. Department of Radiology, The Affiliated Hospital of Qingdao University, Qingdao, Shandong, China; b. Department of Pathology, The Affiliated Hospital of Qingdao University, Qingdao, Shandong, China; c. Department of Radiology, The Third Hospital of Hebei Medical University, Shi jiazhuang, Hebei, China; d. Department of Research Collaboration, R\&D center, Beijing Deepwise \& League of PHD Technology Co, Ltd, Beijing, China; e. Department of Sports Medicine, the Affiliated Hospital of Qingdao University, QingDao, Shandong, China

\section{References}

1. Schuetze SM: Imaging and response in soft tissue sarcomas. Hematol Oncol Clin North Am 2005, 19:471-487, vi.

2. Clark MA, Fisher C, Judson I, Thomas JM: Soft-tissue sarcomas in adults.N Engl J Med 2005, 353:701-711.

3. Burningham Z, Hashibe M, Spector L, Schiffman JD: The epidemiology of sarcoma.Clin Sarcoma Res 2012, 2:14.

4. Bleloch JS, Ballim RD, Kimani S, Parkes J, Panieri E, Willmer T, Prince S: Managing sarcoma: where have we come from and where are we going? Ther Adv Med Oncol 2017, 9:637-659.

5. Kasper B, Gil T, Awada A: Treatment of patients with advanced soft tissue sarcoma: disappointment or challenge? Curr Opin Oncol 2007, 19:336-340.

6. Crombé A, Marcellin PJ, Buy X, Stoeckle E, Brouste V, Italiano A, Le Loarer F, Kind M: Soft-Tissue Sarcomas: Assessment of MRI Features Correlating with Histologic Grade and Patient Outcome.Radiology 2019, 291:710-721.

7. Coindre JM: Grading of soft tissue sarcomas: review and update.Arch Pathol Lab Med 2006, 130:1448-1453.

8. Gronchi A, Ferrari S, Quagliuolo V, Broto JM, Pousa AL, Grignani G, Basso U, Blay JY, Tendero O, Beveridge RD, et al: Histotype-tailored neoadjuvant chemotherapy versus standard chemotherapy in patients with high-risk soft-tissue sarcomas (ISG-STS 1001): an international, open-label, randomised, controlled, phase 3, multicentre trial.Lancet Oncol 2017, 18:812-822.

9. Italiano A, Delva F, Mathoulin-Pelissier S, Le Cesne A, Bonvalot S, Terrier P, Trassard M, Michels JJ, Blay JY, Coindre JM, Bui B: Effect of adjuvant chemotherapy on survival in FNCLCC grade 3 soft tissue sarcomas: a multivariate analysis of the French Sarcoma Group Database.Ann Oncol 2010, 21:2436-2441.

10. Saponara M, Stacchiotti S, Casali PG, Gronchi A: (Neo)adjuvant treatment in localised soft tissue sarcoma: The unsolved affair.Eur J Cancer 2017, 70:1-11.

11. Garcia del Muro X, de Alava E, Artigas V, Bague S, Braña A, Cubedo R, Cruz J, Mulet-Margalef N, Narvaez JA, Martinez Tirado $O$, et al: Clinical practice guidelines for the diagnosis and treatment of patients with soft tissue sarcoma by the Spanish group for research in sarcomas (GEIS). Cancer Chemother Pharmacol 2016, 77:133-146.

12. Schneider N, Strauss DC, Smith MJ, Miah AB, Zaidi S, Benson C, van Houdt WJ, Jones RL, Hayes AJ, Fisher C, Thway K: The Adequacy of Core Biopsy in the Assessment of Smooth Muscle Neoplasms of Soft Tissues: Implications for Treatment and Prognosis.Am J Surg Pathol 2017, 41:923-931. 
13. Liu QY, Li HG, Chen JY, Liang BL: [Correlation of MRI features to histopathologic grade of soft tissue sarcoma].Ai Zheng 2008, 27:856-860.

14. Zhao F, Ahlawat S, Farahani SJ, Weber KL, Montgomery EA, Carrino JA, Fayad LM: Can MR imaging be used to predict tumor grade in soft-tissue sarcoma? Radiology 2014, 272:192-201.

15. Lambin P, Rios-Velazquez E, Leijenaar R, Carvalho S, van Stiphout RG, Granton P, Zegers CM, Gillies R, Boellard R, Dekker A, Aerts HJ: Radiomics: extracting more information from medical images using advanced feature analysis. Eur $J$ Cancer 2012, 48:441-446.

16. Gillies RJ, Kinahan PE, Hricak H: Radiomics: Images Are More than Pictures, They Are Data.Radiology 2016, 278:563-577.

17. Zhang Y, Zhu Y, Shi X, Tao J, Cui J, Dai Y, Zheng M, Wang S: Soft Tissue Sarcomas: Preoperative Predictive Histopathological Grading Based on Radiomics of MRI.Acad Radiol 2019, 26:1262-1268.

18. Wang H, Chen H, Duan S, Hao D, Liu J: Radiomics and Machine Learning With Multiparametric Preoperative MRI May Accurately Predict the Histopathological Grades of Soft Tissue Sarcomas.J Magn Reson Imaging 2020, 51:791-797.

19. Tagliafico AS, Bignotti B, Rossi F, Valdora F, Martinoli C: Local recurrence of soft tissue sarcoma: a radiomic analysis. Radiol Oncol 2019, 53:300-306.

20. Spraker MB, Wootton LS, Hippe DS, Ball KC, Peeken JC, Macomber MW, Chapman TR, Hoff MN, Kim EY, Pollack SM, et al: MRI Radiomic Features Are Independently Associated With Overall Survival in Soft Tissue Sarcoma.Adv Radiat Oncol 2019, 4:413-421.

21. Vallières $M$, Freeman $C R$, Skamene SR, El Naqa I: A radiomics model from joint FDG-PET and MRI texture features for the prediction of lung metastases in soft-tissue sarcomas of the extremities.Phys Med Biol 2015, 60:5471-5496.

22. Pasquali S, Gronchi A: Neoadjuvant chemotherapy in soft tissue sarcomas: latest evidence and clinical implications. Ther Adv Med Oncol 2017, 9:415-429.

23. Depeursinge A, Foncubierta-Rodriguez A, Van De Ville D, Müller H: Threedimensional solid texture analysis in biomedical imaging: review and opportunities.Med Image Anal 2014, 18:176-196.

24. Gibbs P, Turnbull LW: Textural analysis of contrastenhanced MR images of the breast.Magn Reson Med 2003, 50:92-98.

25. Fortin JP, Cullen N, Sheline YI, Taylor WD, Aselcioglu I, Cook PA, Adams P, Cooper C, Fava M, McGrath PJ, et al: Harmonization of cortical thickness measurements across scanners and sites.Neuroimage 2018, 167:104-120.

26. Orlhac F, Frouin F, Nioche C, Ayache N, Buvat I: Validation of A Method to Compensate Multicenter Effects Affecting CT Radiomics.Radiology 2019, 291:53-59.

27. Zhang P, Feng Z, Cai W, You H, Fan C, Lv W, Min X, Wang L: T2-Weighted Image-Based Radiomics Signature for Discriminating Between Seminomas and Nonseminoma.Front Oncol 2019, 9:1330.

28. Balachandran VP, Gonen M, Smith JJ, DeMatteo RP: Nomograms in oncology: more than meets the eye.Lancet Onco/2015, 16:e173-180.

29. Kramer AA, Zimmerman JE: Assessing the calibration of mortality benchmarks in critical care: The Hosmer-Lemeshow test revisited.Crit Care Med 2007, 35:2052-2056.

30. Brinkmann EJ, Ahmed SK, Houdek MT: Extremity Soft Tissue Sarcoma: Role of Local Control.Curr Treat Options Oncol 2020, 21:13.

31. Peeken JC, Bernhofer M, Wiestler B, Goldberg T, Cremers D, Rost B, Wilkens JJ, Combs SE, Nüsslin F: Radiomics in radiooncology - Challenging the medical physicist.Phys Med 2018, 48:27-36.

32. Peeken JC, Hesse J, Haller B, Kessel KA, Nüsslin F, Combs SE: Semantic imaging features predict disease progression and survival in glioblastoma multiforme patients.Strahlenther Onkol 2018, 194:580-590.

33. Peeken JC, Nüsslin F, Combs SE: "Radio-oncomics" : The potential of radiomics in radiation oncology.Strahlenther Onkol 2017, 193:767-779.

34. Corino VDA, Montin E, Messina A, Casali PG, Gronchi A, Marchianò A, Mainardi LT: Radiomic analysis of soft tissues sarcomas can distinguish intermediate from high-grade lesions.J Magn Reson Imaging 2018, 47:829-840. 
35. Xiang P, Zhang X, Liu D, Wang C, Ding L, Wang F, Zhang Z: Distinguishing soft tissue sarcomas of different histologic grades based on quantitative MR assessment of intratumoral heterogeneity. Eur J Radiol 2019, 118:194-199.

36. Yi X, Pei Q, Zhang Y, Zhu H, Wang Z, Chen C, Li Q, Long X, Tan F, Zhou Z, et al: MRI-Based Radiomics Predicts Tumor Response to Neoadjuvant Chemoradiotherapy in Locally Advanced Rectal Cancer.Front Oncol 2019, 9:552.

37. Peeken JC, Spraker MB, Knebel C, Dapper H, Pfeiffer D, Devecka M, Thamer A, Shouman MA, Ott A, von Eisenhart-Rothe R, et al: Tumor grading of soft tissue sarcomas using MRI-based radiomics.EBioMedicine 2019, 48:332-340.

38. Wang H, Nie P, Wang Y, Xu W, Duan S, Chen H, Hao D, Liu J: Radiomics nomogram for differentiating between benign and malignant soft-tissue masses of the extremities.J Magn Reson Imaging 2020, 51:155-163.

39. Italiano A, Le Cesne A, Mendiboure J, Blay JY, Piperno-Neumann S, Chevreau C, Delcambre C, Penel N, Terrier P, RanchereVince D, et al: Prognostic factors and impact of adjuvant treatments on local and metastatic relapse of soft-tissue sarcoma patients in the competing risks setting.Cancer 2014, 120:3361-3369.

40. Wilson DAJ, Gazendam A, Visgauss J, Perrin D, Griffin AM, Chung PW, Catton CN, Shultz D, Ferguson PC, Wunder JS: Designing a Rational Follow-Up Schedule for Patients with Extremity Soft Tissue Sarcoma.Ann Surg Oncol 2020.

\section{Tables}




\begin{tabular}{|c|c|c|}
\hline & \multicolumn{2}{|l|}{ Number of Patients } \\
\hline & Training set $(n=109)$ & External validation set $(n=71)$ \\
\hline liposarcoma & 17 & 16 \\
\hline Undifferentiated pleomorphic sarcoma & 23 & 7 \\
\hline Myxofibrosarcoma & 13 & 8 \\
\hline Synovial sarcoma & 11 & 9 \\
\hline Fibrosarcoma & 6 & 4 \\
\hline Leiomyosarcoma & 6 & 3 \\
\hline Rhabdomyosarcoma & 5 & 5 \\
\hline Extraskeletal chondrosarcoma & 5 & 4 \\
\hline Undifferentiated sarcoma & 2 & 4 \\
\hline Myxoinflammatory fibroblastic sarcoma & 0 & 2 \\
\hline Clear cell sarcoma & 2 & 1 \\
\hline Spindle cell sarcoma & 4 & 0 \\
\hline Myofibroblastic sarcoma & 1 & 1 \\
\hline Alveolar soft part sarcoma & 1 & 1 \\
\hline Desmoid fibromatosis & 1 & 1 \\
\hline Angiosarcoma & 0 & 2 \\
\hline Malignant peripheral nervesheath tumor & 2 & 0 \\
\hline Extraskeletal Ewing Sarcoma & 0 & 1 \\
\hline Primitive neuroectodermal tumor & 2 & 0 \\
\hline Epithelioid hemangioendothelioma & 0 & 1 \\
\hline Malignant solitary fibrous tumor & 2 & 0 \\
\hline malignant melanoma & 0 & 1 \\
\hline Kaposi sarcoma & 1 & 0 \\
\hline Not Otherwise Specified & 5 & 0 \\
\hline
\end{tabular}




\begin{tabular}{|c|c|c|c|c|c|c|}
\hline & \multicolumn{3}{|c|}{ Training set $(n=109)$} & \multicolumn{3}{|c|}{ External validation set $(n=71)$} \\
\hline & $\begin{array}{l}\text { Low }^{a}{ }^{-} \\
(n=59)\end{array}$ & $\begin{array}{l}\text { High- }^{a} \\
(n=50)\end{array}$ & $P^{*}$ & $\begin{array}{l}L{ }^{a}{ }^{a} \\
(n=34)\end{array}$ & $\begin{array}{l}\text { High- }^{a} \\
(n=37)\end{array}$ & $P^{*}$ \\
\hline Age (years) (mean \pm SD) & $51.0 \pm 20.2$ & $55.0 \pm 19.1$ & 0.312 & $53.3 \pm 19.3$ & $50.4 \pm 18.7$ & 0.521 \\
\hline \multicolumn{7}{|l|}{ Gender } \\
\hline Male & 38 & 33 & \multirow[t]{2}{*}{0.862} & 18 & 23 & \multirow[t]{2}{*}{0.432} \\
\hline Female & 21 & 17 & & 16 & 14 & \\
\hline \multicolumn{7}{|l|}{ T-stage ${ }^{b}$} \\
\hline 1 & 17 & 9 & \multirow[t]{4}{*}{0.003} & 9 & 4 & \multirow[t]{4}{*}{0.001} \\
\hline 2 & 31 & 19 & & 23 & 16 & \\
\hline 3 & 10 & 10 & & 1 & 9 & \\
\hline 4 & 1 & 12 & & 1 & 8 & \\
\hline \multicolumn{7}{|l|}{$\mathrm{N}$-stage $\mathrm{e}^{\mathrm{b}}$} \\
\hline 0 & 58 & 49 & \multirow[t]{2}{*}{1.000} & 34 & 35 & \multirow[t]{2}{*}{0.494} \\
\hline 1 & 1 & 1 & & 0 & 2 & \\
\hline \multicolumn{7}{|l|}{ M-stage ${ }^{b}$} \\
\hline 0 & 57 & 40 & \multirow[t]{2}{*}{0.006} & 32 & 32 & \multirow[t]{2}{*}{0.432} \\
\hline 1 & 2 & 10 & & 2 & 5 & \\
\hline \multicolumn{7}{|l|}{ AJCC-stage } \\
\hline $\mathrm{IA}$ & 1 & 3 & \multirow[t]{6}{*}{0.005} & 0 & 0 & \multirow[t]{6}{*}{0.351} \\
\hline IB & 15 & 2 & & 6 & 3 & \\
\hline$\| \mathrm{A}$ & 14 & 9 & & 7 & 6 & \\
\hline IIB & 18 & 15 & & 12 & 22 & \\
\hline III & 8 & 11 & & 5 & 3 & \\
\hline IV & 3 & 10 & & 4 & 3 & \\
\hline Median PFS & 43 & 19 & 0.000 & 39 & 24 & 0.002 \\
\hline \multicolumn{7}{|l|}{ MRI features } \\
\hline \multicolumn{7}{|l|}{$\mathrm{T} 1$ high signal matrix } \\
\hline+ & 22 & 18 & \multirow[t]{2}{*}{0.889} & 20 & 13 & \multirow[t]{2}{*}{0.046} \\
\hline- & 37 & 32 & & 14 & 24 & \\
\hline \multicolumn{7}{|l|}{ T2 low signal matrix } \\
\hline+ & 25 & 23 & \multirow[t]{2}{*}{0.704} & 30 & 31 & \multirow[t]{2}{*}{0.737} \\
\hline- & 34 & 27 & & 4 & 6 & \\
\hline
\end{tabular}




\begin{tabular}{|c|c|c|c|c|c|c|}
\hline+ & 33 & 34 & \multirow[t]{2}{*}{0.197} & 19 & 23 & \multirow[t]{2}{*}{0.591} \\
\hline- & 26 & 16 & & 15 & 14 & \\
\hline \multicolumn{7}{|c|}{ Myxold matrix } \\
\hline+ & 18 & 7 & \multirow[t]{2}{*}{0.041} & 18 & 18 & \multirow[t]{2}{*}{0.718} \\
\hline- & 41 & 43 & & 16 & 19 & \\
\hline \multicolumn{7}{|c|}{ Fibrous tissue signal } \\
\hline+ & 10 & 9 & \multirow[t]{2}{*}{0.885} & 30 & 22 & \multirow[t]{2}{*}{0.006} \\
\hline- & 49 & 41 & & 4 & 15 & \\
\hline \multicolumn{7}{|l|}{ Margin } \\
\hline ill-defined & 13 & 32 & \multirow[t]{2}{*}{0.000} & 7 & 26 & \multirow[t]{2}{*}{0.000} \\
\hline well-defined & 46 & 18 & & 27 & 11 & \\
\hline \multicolumn{7}{|l|}{ Septations } \\
\hline+ & 17 & 6 & \multirow[t]{2}{*}{0.032} & 24 & 21 & \multirow[t]{2}{*}{0.227} \\
\hline- & 42 & 44 & & 10 & 16 & \\
\hline \multicolumn{7}{|c|}{ Fat tissue signal } \\
\hline+ & 23 & 20 & \multirow[t]{2}{*}{0.914} & 16 & 12 & \multirow[t]{2}{*}{0.208} \\
\hline- & 36 & 30 & & 18 & 25 & \\
\hline \multicolumn{7}{|l|}{ Vessels } \\
\hline+ & 7 & 3 & \multirow[t]{2}{*}{0.338} & 16 & 22 & \multirow[t]{2}{*}{0.295} \\
\hline- & 52 & 47 & & 18 & 15 & \\
\hline \multicolumn{7}{|l|}{ Hemorrhage } \\
\hline+ & 6 & 4 & \multirow[t]{2}{*}{0.751} & 2 & 3 & \multirow[t]{2}{*}{1.000} \\
\hline- & 53 & 46 & & 32 & 34 & \\
\hline \multicolumn{7}{|c|}{ Maximal depth $>8 \mathrm{~cm}$} \\
\hline+ & 32 & 28 & \multirow[t]{2}{*}{0.854} & 20 & 19 & \multirow[t]{2}{*}{0.527} \\
\hline- & 27 & 22 & & 14 & 18 & \\
\hline \multicolumn{7}{|c|}{ Peritumoral edema } \\
\hline+ & 8 & 13 & \multirow[t]{2}{*}{0.101} & 19 & 23 & 0.591 \\
\hline- & 51 & 37 & & 15 & 14 & \\
\hline Bone involver & & & & & & \\
\hline+ & 10 & 7 & 0.672 & 1 & 8 & 0.029 \\
\hline- & 49 & 43 & & 33 & 29 & \\
\hline Capsule & & & & & & \\
\hline+ & 22 & 26 & 0.123 & 14 & 14 & 0.774 \\
\hline- & 37 & 24 & & 20 & 23 & \\
\hline
\end{tabular}




\begin{tabular}{|c|c|c|c|c|c|c|}
\hline+ & 5 & 4 & \multirow[t]{2}{*}{0.276} & 16 & 25 & \multirow[t]{2}{*}{0.081} \\
\hline - & 54 & 46 & & 18 & 12 & \\
\hline
\end{tabular}

Abbreviation: SD standard deviation

a Grade III are defined as high grade. Grade I and II are defined as low grade.

b TNM stage were accessed from preoperative MRI and computed tomography information.

* Calculated from student $\mathrm{t}$ test or Mann-Whitney $\mathrm{U}$ test for continuous variables and chi-square test or Fisher exact test for classified variables, where appropriate.

\begin{tabular}{|c|c|c|c|c|c|c|}
\hline \multirow[b]{2}{*}{ Variable } & \multicolumn{3}{|c|}{ Univariate logistic analysis } & \multicolumn{3}{|c|}{ Multivariate logistic analysis } \\
\hline & OR & $(95 \% \mathrm{Cl})$ & $\mathrm{P}$ & OR & $(95 \% \mathrm{Cl})$ & $\mathrm{P}$ \\
\hline Radscore & 37.88 & [7.71;435.29] & $<0.001$ & 7.20 & [3.37;19.04] & $<0.001$ \\
\hline T-stage & 3.67 & {$[1.38 ; 11.17]$} & 0.013 & 1.81 & {$[1.14 ; 2.95]$} & 0.026 \\
\hline Myxoid matrix & 0.27 & {$[0.07 ; 0.96]$} & 0.049 & & & \\
\hline Margin & 5.78 & {$[1.66 ; 24.05]$} & 0.009 & 2.69 & {$[1.21 ; 6.19]$} & $<0.001$ \\
\hline Capsule & 7.16 & {$[2.03 ; 31.56]$} & 0.004 & & & \\
\hline
\end{tabular}

Abbreviations: OR: odds ratio, Cl: confidence interval. 


\begin{tabular}{|c|c|c|c|c|c|c|c|c|c|c|c|c|}
\hline & \multicolumn{6}{|c|}{ Training set } & \multicolumn{6}{|c|}{ External validation set } \\
\hline & AUC & $\mathrm{ACC}$ & SEN & SPE & PPV & NPV & AUC & $\mathrm{ACC}$ & SEN & SPE & PPV & NPV \\
\hline & $(95 \% \mathrm{Cl})$ & & & & & & $(95 \% \mathrm{Cl})$ & & & & & \\
\hline \multirow{2}{*}{$\begin{array}{l}\text { Clinical } \\
\text { model }\end{array}$} & 0.787 & 0.770 & 0.680 & 0.847 & 0.791 & 0.758 & 0.833 & 0.761 & 0.676 & 0.853 & 0.833 & 0.707 \\
\hline & $\begin{array}{l}(0.697- \\
0.877)\end{array}$ & & & & & & $\begin{array}{l}(0.783- \\
0.927)\end{array}$ & & & & & \\
\hline \multirow[t]{2}{*}{ RS-T1 } & 0.787 & 0.725 & 0.720 & 0.729 & 0.692 & 0.754 & 0.645 & 0.718 & 0.757 & 0.676 & 0.718 & 0.719 \\
\hline & $\begin{array}{l}(0.703- \\
0.870)\end{array}$ & & & & & & $\begin{array}{l}(0.514- \\
0.777)\end{array}$ & & & & & \\
\hline \multirow[t]{2}{*}{ RS-FST2 } & 0.680 & 0.752 & 0.820 & 0.695 & 0.695 & 0.820 & 0.641 & 0.732 & 0.757 & 0.706 & 0.737 & 0.727 \\
\hline & $\begin{array}{l}(0.579- \\
0.780)\end{array}$ & & & & & & $\begin{array}{l}(0.511- \\
0.771)\end{array}$ & & & & & \\
\hline \multirow{2}{*}{$\begin{array}{l}\text { RS- } \\
\text { Combined }\end{array}$} & 0.846 & 0.761 & 0.740 & 0.780 & 0.740 & 0.780 & 0.829 & 0.789 & 0.676 & 0.912 & 0.893 & 0.769 \\
\hline & $\begin{array}{l}(0.773- \\
0.918)\end{array}$ & & & & & & $\begin{array}{l}(0.715- \\
0.943)\end{array}$ & & & & & \\
\hline \multirow{2}{*}{$\begin{array}{l}\text { Radiomics } \\
\text { nomogram }\end{array}$} & 0.916 & 0.826 & 0.780 & 0.864 & 0.830 & 0.823 & 0.879 & 0.817 & 0.838 & 0.794 & 0.816 & 0.818 \\
\hline & $\begin{array}{l}(0.866- \\
0.966)\end{array}$ & & & & & & $\begin{array}{l}(0.791- \\
0.967)\end{array}$ & & & & & \\
\hline
\end{tabular}

Abbreviations: AUC: area under curve, ACC: accuracy, SEN: sensitivity, SPE: specificity, PPV: positive predictive value, NPV: negative predictive value.

Figures 


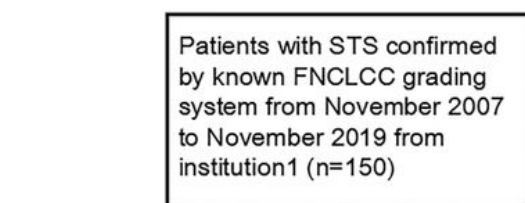

Patients with STS confirmed by known FNCLCC grading system from November 2007 to November 2019 from institution2 $(n=98)$

\begin{tabular}{|l|}
25 patients excluded \\
- unavailable or \\
incomplete relevant \\
clinical or MRI data \\
— images with a low \\
signal-to-noise ratio \\
$(\leq 1.0)$
\end{tabular}

13 patients excluded - not complete pathological data or

MRI images information — low signal-to-noise ratio (SNR) ( $\leq 1.0)$

images

\begin{tabular}{|l|}
16 patients excluded \\
- performance of \\
treatment \\
- development of \\
other unrelated tumors
\end{tabular}

14 patients excluded - performance of treatment

- development of

other unrelated tumors

The training set $(n=109)$

Model construction

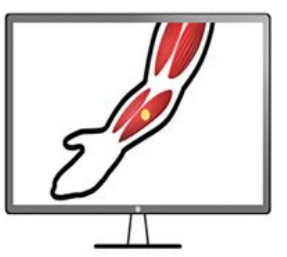

T1WI, FS-T2WI

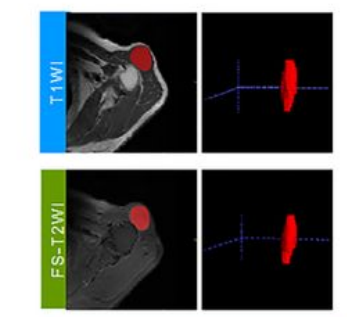

Normalized

using cubic interpolation method
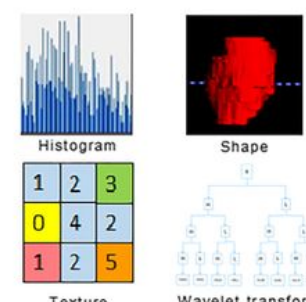

Shape

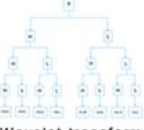

voxel size

mage preprocessing

\section{Feature extraction}

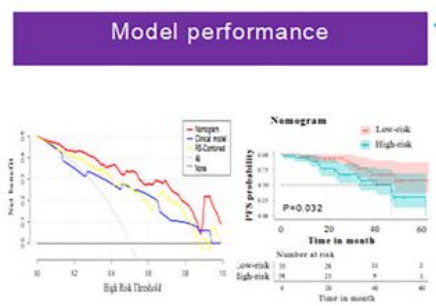

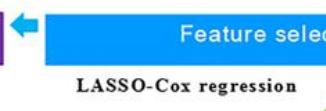

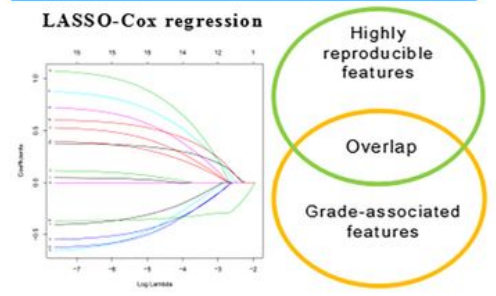

Multicenter standardization method

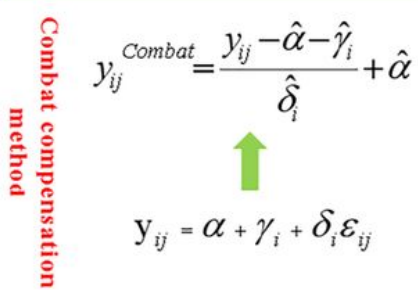

Figure 1

(Top) Flow chart of the enrolled patients. FNCLCC: French Federation of Cancer Centers Sarcoma Group. (Bottom) Flow chart of the radiomics implementation of this study. LASSO: least absolute shrinkage and selection operator. 

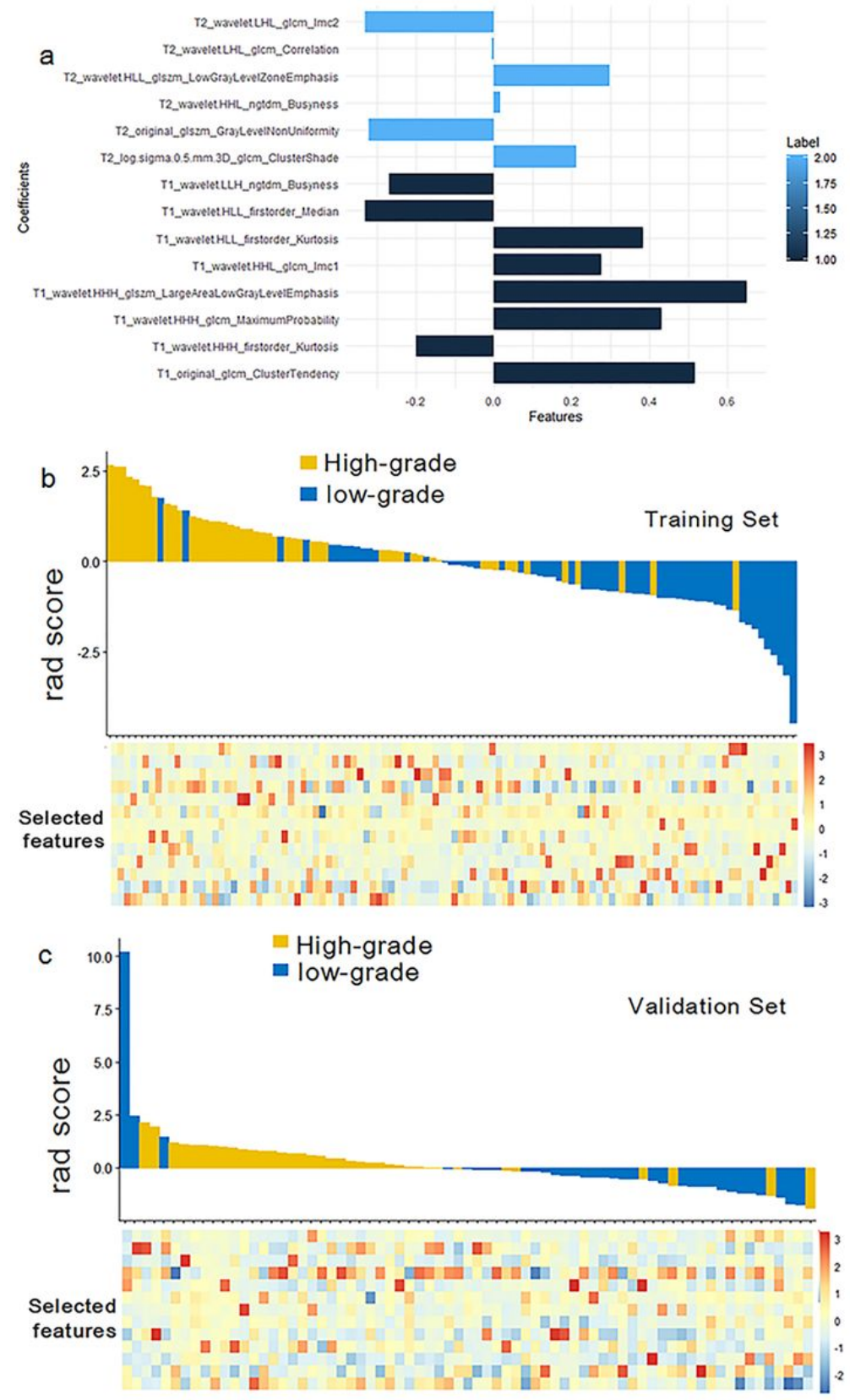

\section{Figure 2}

(a) The 14 individual features with accordingly non-zero coefficients that contributed to the developed RS-Combined model. (b,

c) The radiomics score (rad-score) of each patient in the training set and external validation set separately. 

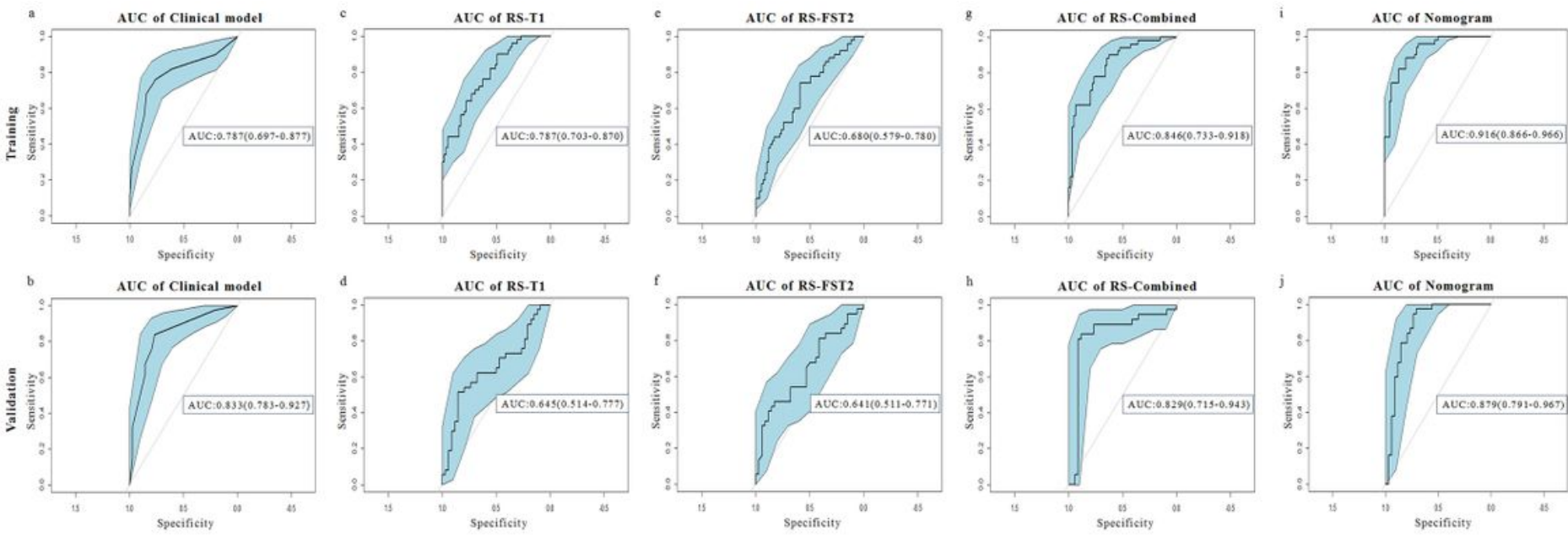

Figure 3

Receiver operating characteristic curves of (a, b) Clinical model, (c, d) RS-T1 model, (e, f) RS-FST2 model, (g, h) RS-Combined model, and $(\mathrm{i}, \mathrm{j})$ radiomics nomogram model for predicting the histopathological grade in the training set and validation set separately. The shaded blue parts show the $95 \%$ confidence interval of the area under the curve and is depicted within brackets.

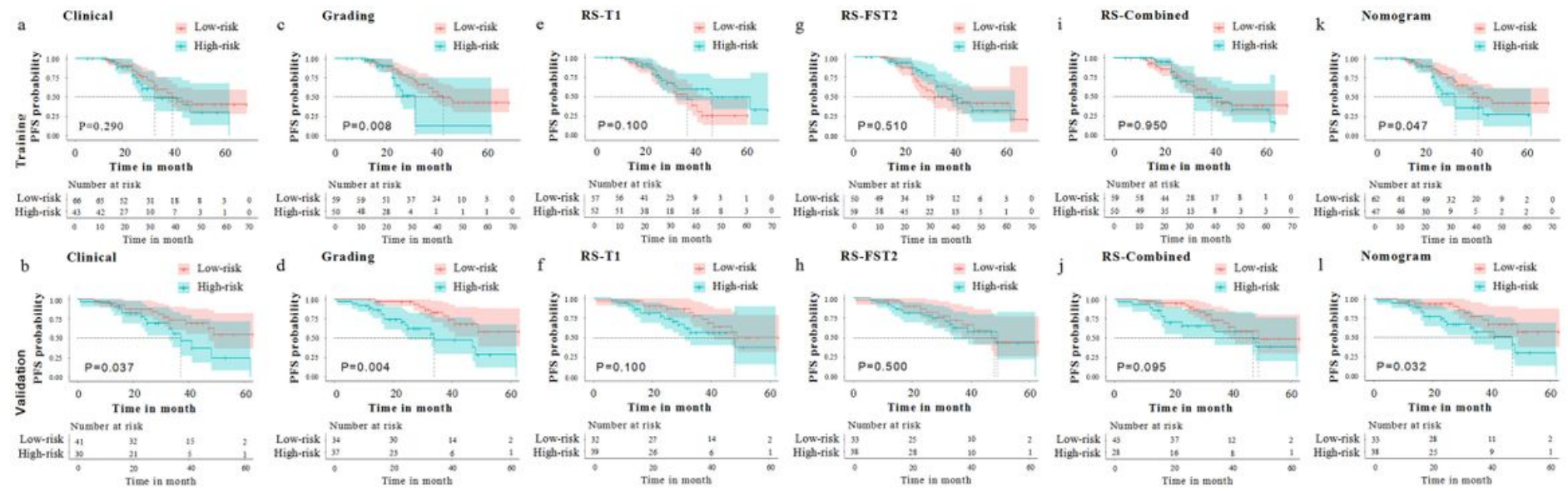

\section{Figure 4}

Results of Kaplan-Meier survival curves for progression-free survival of the $(a, b)$ Clinical model, $(c, d)$ tumor grading model (low-grade vs. high-grade), (e, f) RS-T1 model, ( $g, h)$ RS-FST2 model, ( $i, j)$ RS-Combined model, and ( $k$, l) radiomics nomogram model in the training set and external validation set separately. 
a

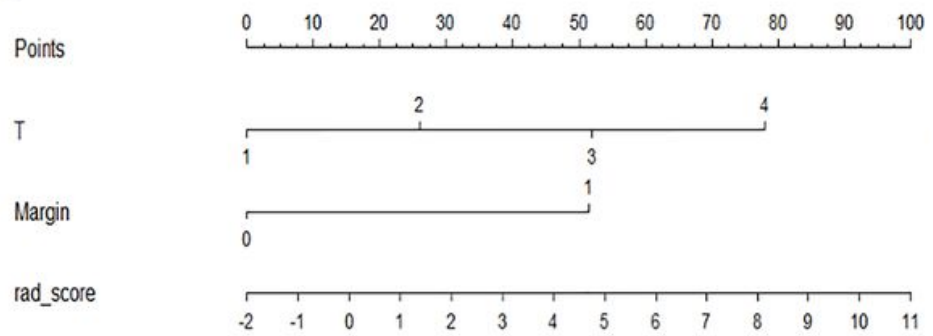

Total Points

Risk $\mathrm{b}$
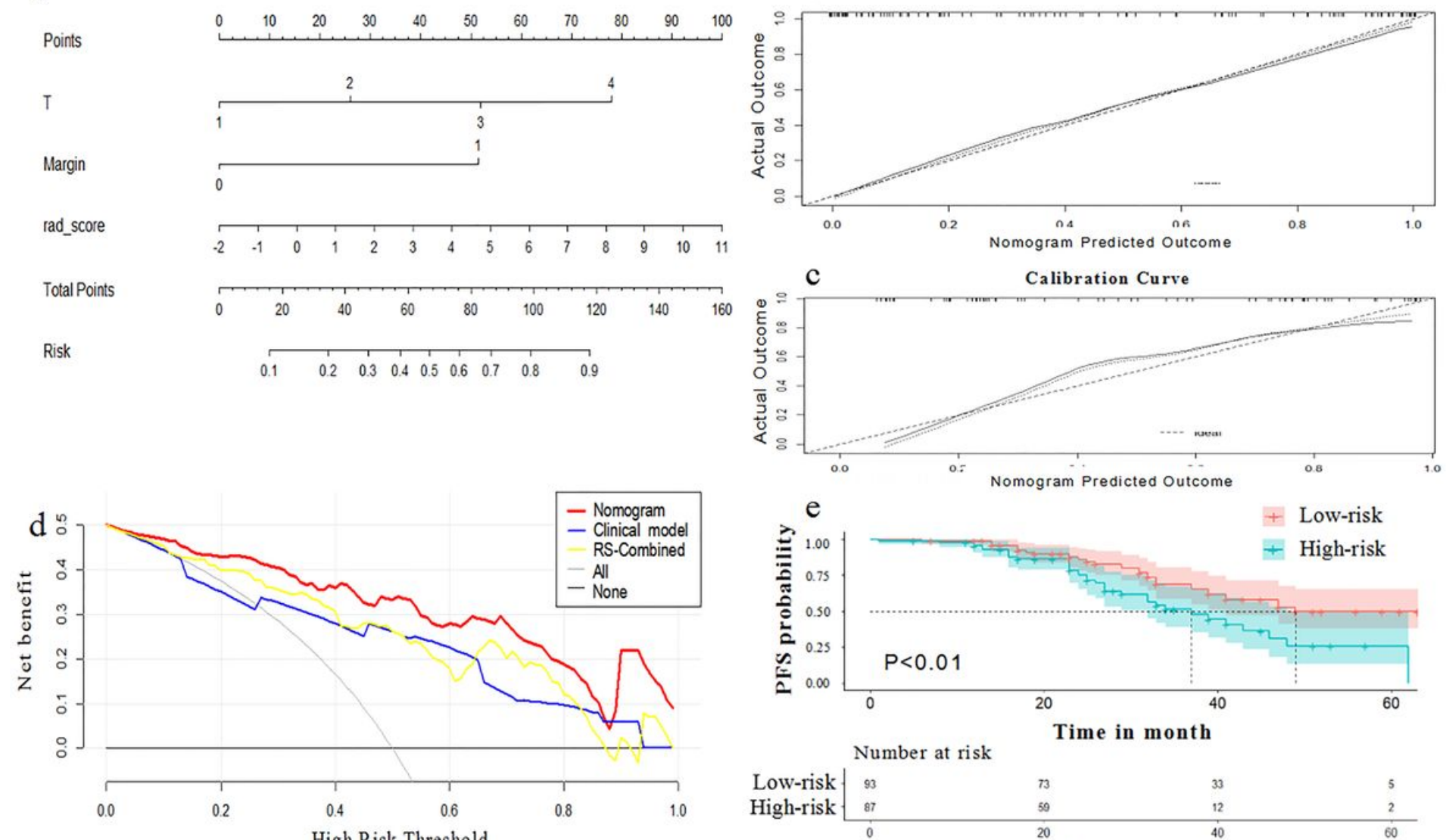

High Risk Threshold

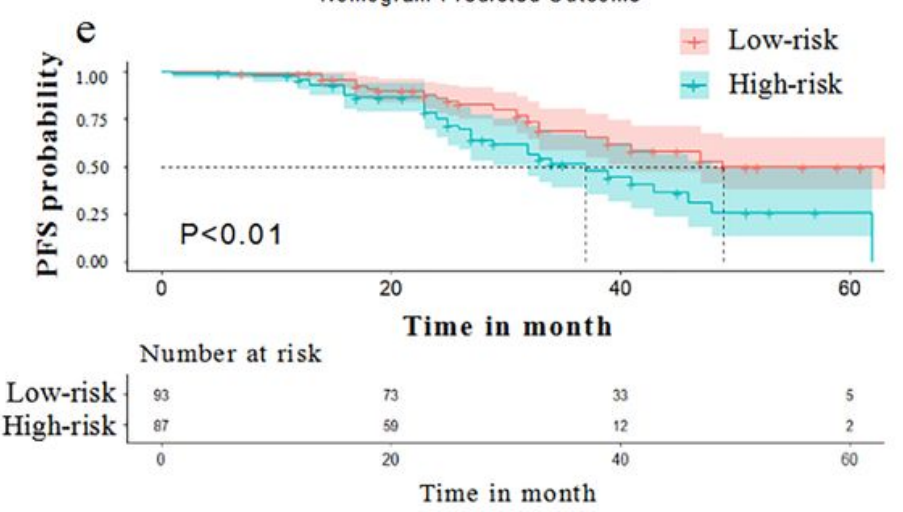

Figure 5

(a) Radiomics nomogram for forecasting preoperative classification of patients with soft tissue sarcoma based on combination of the RS-Combined model and clinical factors. (b, c) Calibration curves of radiomics nomogram in the training set and validation set. (d) Decision curve analysis for the radiomics nomogram. (e) The Kaplan-Meier survival curve for progressionfree survival of the proposed nomogram combined with the AJCC staging system is depicted

\section{Supplementary Files}

This is a list of supplementary files associated with this preprint. Click to download.

- Additionalfile1.docx 